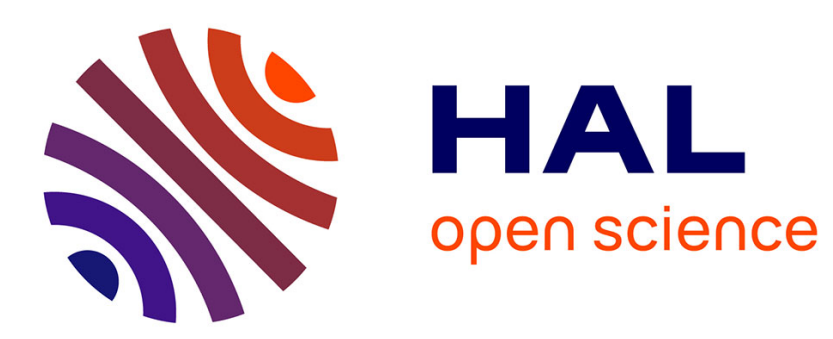

\title{
Diffractive optical elements in hybrid lenses: modeling and design by zone decomposition
}

Hervé Sauer, Pierre Chavel, Gabor Erdei

\section{To cite this version:}

Hervé Sauer, Pierre Chavel, Gabor Erdei. Diffractive optical elements in hybrid lenses: modeling and design by zone decomposition. Applied optics, 1999, 38 (31), pp.6482-6486. hal-00867851

\section{HAL Id: hal-00867851 \\ https://hal-iogs.archives-ouvertes.fr/hal-00867851}

Submitted on 30 Sep 2013

HAL is a multi-disciplinary open access archive for the deposit and dissemination of scientific research documents, whether they are published or not. The documents may come from teaching and research institutions in France or abroad, or from public or private research centers.
L'archive ouverte pluridisciplinaire HAL, est destinée au dépôt et à la diffusion de documents scientifiques de niveau recherche, publiés ou non, émanant des établissements d'enseignement et de recherche français ou étrangers, des laboratoires publics ou privés. 


\title{
Diffractive optical elements in hybrid lenses: modeling and design by zone decomposition
}

\author{
Hervé Sauer, Pierre Chavel, and Gábor Erdei
}

\begin{abstract}
We propose to model hybrid optical systems (i.e., lenses with conventional and diffractive optical elements) as multiaperture systems in which the images formed by each zone of the diffractive optical element should be summed up coherently. This new zone decomposition concept is explained and compared with the standard diffraction-order expansion with the help of a hybrid triplet example. (C) 1999 Optical Society of America

OCIS codes: $\quad 050.1970,220.3620,110.3000,110.4100$.
\end{abstract}

\section{Introduction}

We discuss the computation of the point-spread function (PSF) and the modulation transfer function (MTF) of hybrid lenses, i.e., optical imaging systems that combine conventional (refractive or reflective) surfaces and diffractive optical elements (DOE's). Such hybrid systems offer new attractive solutions to some optical design problems; thanks to progress in manufacturing techniques, they have been used in IR and visible band for a few years. ${ }^{1-3}$ However, standard design tools suffer from limitation in their optical-quality analysis. In particular, most commercial software packages for optical design model and design the system with only one diffractive order of the DOE, the so-called nominal order-in most cases the \pm 1 order. This generates errors that are noticeable as soon as a significant fraction of the light is diffracted into the other orders. The effect is well known. One rough corrective action has been customary, although it is not, as far as we know, implemented as such in commercial software: nonnominal orders are usually significantly defocused and participate in the image formation mostly through a uniform background that can be accounted for as a

\footnotetext{
When this research was performed, the authors were with Laboratoire Charles Fabry, Institut d'Optique Théorique et Appliquée, Centre National de la Recherche Scientifique, Unité Mixte de Recherche 8501-Centre Universitaire, Bâtiment 503, B. P. 147-91403, Orsay Cedex France. G. Erdei is now with the Department of Atomic Physics, Optical Research Laboratory, Technical University of Budapest, H-1111 Budapest, Budafoki út 8., Hungary. H. Sauer's e-mail address is herve.sauer@iota.u-psud.fr.

Received 26 April 1999; revised manuscript received 12 July 1999.

0003-6935/99/316482-05\$15.00/0

(C) 1999 Optical Society of America
}

central peak in the MTF; in this approximation, it is sufficient to multiply, at all nonzero spatial frequencies, the nominal-order normalized MTF by the ratio of the useful light power to the total light power (in the monochromatic case, the factor is simply the nominalorder efficiency). ${ }^{4}$ However, this does not account well for the not uncommon case of a small number of zones, where the nonnominal low-order PSF's are only a few times wider than the nominal-order PSF and, therefore, participate in some extended range of spatial frequencies. Moreover, it is impossible with such an approximation to include those orders in the optimization process.

Indeed, infinite summation of orders is a rigorous solution as far as the scalar approximation of optical systems holds; such summation should be performed coherently for all orders at each wavelength. The convergence of the diffraction-orders series expansion, however, is known to be often impractical owing to its slow $1 / M$ convergence, with $M$ being the number of summed-up orders. One may note that incoherent-order summation, sometimes used by optical designers, has the same slow $1 / M$ convergence behavior but leads to a mathematically incorrect answer.

In the following, we propose a simple alternative method based on zone decomposition rather than on order summation. The principle is described in Section 2 , and one illustrative example is presented in Section 3.

\section{Modeling Principle}

First, let us note that in aberration-correction applications, the groove spacing of the diffractive optical elements is usually large compared with the wavelength. Thus, the theoretical background to our 


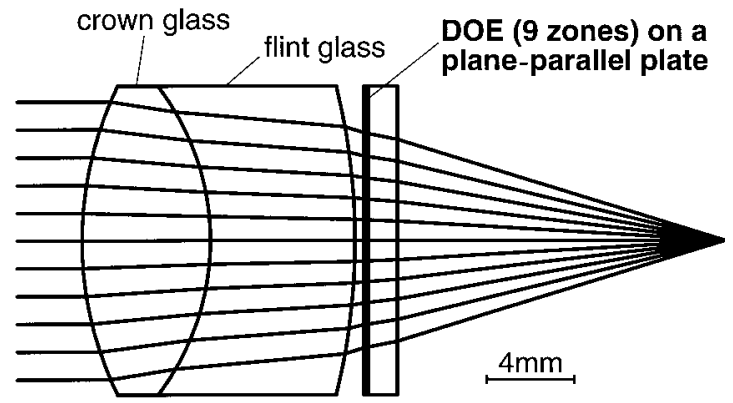

Fig. 1. Layout of the hybrid triplet. This system is an $f / 1.7$, 21.1-mm focal length lens intended to work in the visible and the NIR band $(0.48-0.78 \mu \mathrm{m})$.

computations is the scalar theory of diffraction and mainly the Kirchhoff diffraction integral.5,6 The PSF is then the two-dimensional (2D) Fourier transform of the complex amplitude (relative to the given reference sphere) in the exit pupil of the system. In our proposed method, we model the hybrid system as a multiaperture system, much in the same way as in multipupil telescopes in which the images owing to the various pupils combine coherently into one final image. In the present case, however, the various apertures correspond to the DOE zones: namely, optical thickness jumps partition the DOE surface into nonoverlapping apertures, which, in the common case of rotationally symmetric systems, are concentric annular areas. In fact, the discontinuity between zones is designed to create a phase jump for the outgoing wave front, and the main idea in DOE's is to have $2 \pi$ phase jumps between adjacent zones (or an integer multiple thereof) at least on axis for some nominal wavelength. Within each zone, DOE optical thickness varies continuously like in any standard component in a lens, and then, conventional analysis methods, like ray tracing, can be used there. Note that in the case of the so-called binary optics manufacturing method $^{7}$ for which multiple masking steps are used to approximate, on the outgoing wave front, a continuous phase variation over $2 \pi$ by a $p$-step staircase function, each step defines one subzone with $2 \pi / p$ phase discontinuities. In such a case, the elementary aperture that should be used is the subzone.

The analysis method of the whole hybrid lens is then straightforward: each monochromatic image is the coherent sum of the images given by the system for each zone or each subzone. Any tool, including commercial optical design software, can be used to model the system behavior as soon as diffraction PSF of systems with nonstandard aperture and coherent (complex) summation are available. Let us emphasize that the infinite summation of orders is mathematically equivalent to our, potentially simpler, finite summation based on zone decomposition. Moreover, the zone decomposition still holds when the phase discontinuities do not have the same value at all zone boundaries, even though the order decompo-
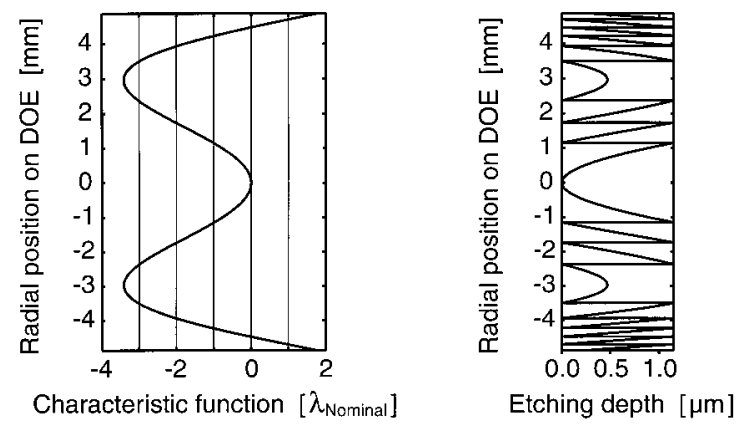

Fig. 2. Characteristic function and etching profile of the hybrid triplet DOE. Note the unusual shape of the characteristic function curve that is mainly intended to correct for spherical aberration (mostly like a Schmidt plate) rather than for pure axial chromatism. $\lambda_{\text {Nominal }}=594 \mathrm{~nm}$.

sition no longer exists from a mathematical point of view.

From the monochromatic PSF of the whole system, obtained as explained above, standard methods apply to derive the monochromatic MTF. As usual, one performs polychromatic analysis by use of incoherent weighted summation over wavelength.

\section{One Example: A Fast Achromatic Hybrid Triplet}

One of the major motivations for using DOE's inside hybrid systems is the dispersive behavior of diffraction, which provides efficient means to deal with chromatic aberration. In the order decomposition method, nonnominal orders essentially create defocused images that, in the absence of any other aberration, result in more or less uniform plateaus in the PSF. Our approach directly leads to the result of interferences between the plateaus of all orders at each wavelength. We already presented such a result $^{8}$ and intend to publish more detailed examples at a later stage. Here, we emphasize the advantage of the proposed method on just one characteristic example for which the DOE corrects for combined aberrations rather than for pure chromatism. Although it

Table 1. Detailed Layout of the Hybrid Triplet

\begin{tabular}{lcccc}
\hline $\begin{array}{c}\text { Surface } \\
\text { Number }\end{array}$ & Surface Type & $\begin{array}{c}\text { Radius of } \\
\text { Curvature } \\
(\mathrm{mm})\end{array}$ & $\begin{array}{c}\text { Thickness } \\
(\mathrm{mm})\end{array}$ & Material \\
\hline 0 (Object) & Flat & Infinity & Infinity & - \\
1 (Stop) & Sphere & 15.94 & 5.80 & SK2 (Schott) \\
2 & Sphere & -11.53 & 6.50 & SF4 (Schott) \\
3 & Sphere & -29.34 & 0.40 & - \\
4 & Flat + DOE & Infinity & 1.50 & BK7 (Schott) \\
5 & Flat & Infinity & 14.70 & - \\
6 (Image) & Flat & Infinity & - & - \\
\hline
\end{tabular}

aThe specifications for the DOE were as follows: $\quad \lambda_{\text {Nominal }}=594$ $\mathrm{nm}$; the nominal diffraction order was +1 ; the characteristic function was a radial polynomial; and the phase at $\lambda_{\text {Nominal }}$ was $2 \pi / \lambda_{\text {Nominal }}\left[\left(c_{1} h^{2}\right)+\left(c_{2} h^{4}\right)+\left(c_{3} h^{6}\right)+\left(c_{4} h^{8}\right)\right]$, where $c_{1}=$ $-4.903 \times 10^{-4} \mathrm{~mm}^{-1}, c_{2}=3.263 \times 10^{-5} \mathrm{~mm}^{-3}, c_{3}=-3.012 \times$ $10^{-7} \mathrm{~mm}^{-5}, c_{4}=-5.268 \times 10^{-9} \mathrm{~mm}^{-7}$, and $h$ is the radial distance from the optical axis. 


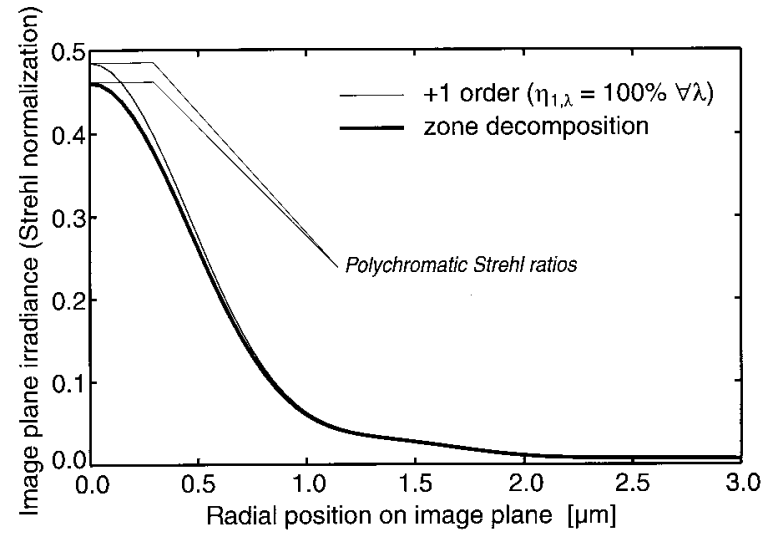

Fig. 3. On-axis polychromatic PSF of the hybrid triplet (central region). Thin line: +1 diffraction-order computation (100\% efficiency assumed for all wavelengths). Thick line: zonedecomposition computation.

may not correspond to a very practical case in view of some manufacturing constraints, this example is intended to illustrate our point of view about the coherent multiaperture image summation.

Figure 1 sketches the system layout: It is a visible-near-infrared triplet built from a cemented doublet and a nine-zone DOE with continuous zone profiles on a plane-parallel plate. The characteristic function and the etching profile of the DOE are shown on Fig. 2. The precise description can be found in Table 1 . The result is a fairly good $21.1-\mathrm{mm}$ focal length, $f / 1.7$ achromat for infinity-focal-plane conjugation in the wavelength range $0.48-0.78 \mu \mathrm{m} .{ }^{9} \quad$ The polychromatic analyses are done with 11 equispaced wavelengths with equal weight. Figure 3 shows the triplet polychromatic PSF in the immediate vicinity of the axis. The departure between the standard calculation with only one order (with $100 \%$ efficiency at all wavelengths, as assumed by most commercial optical design software) is fairly clear from the Strehl ratio. Figure 4 gives the same PSF in a log scale for a much wider radial extent, showing the jagged as-

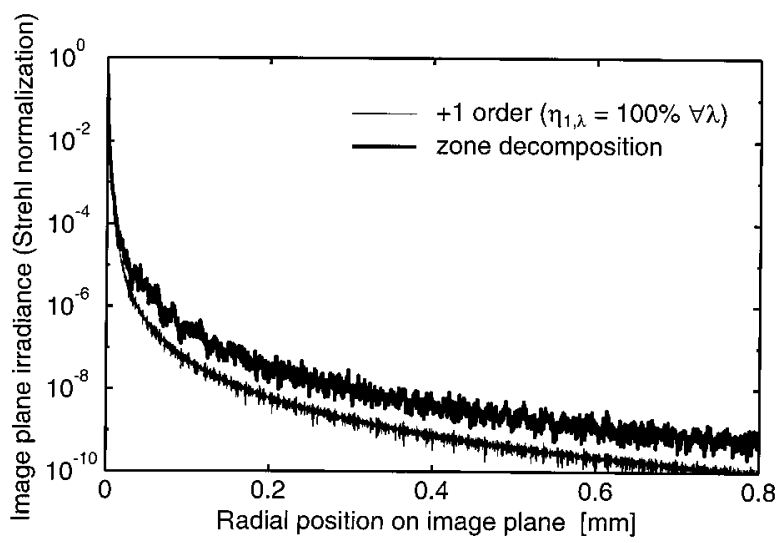

Fig. 4. On-axis polychromatic PSF of the hybrid triplet (wide region). Thin line: +1 diffraction-order computation (100\% efficiency assumed for all wavelengths). Thick line: zonedecomposition computation.

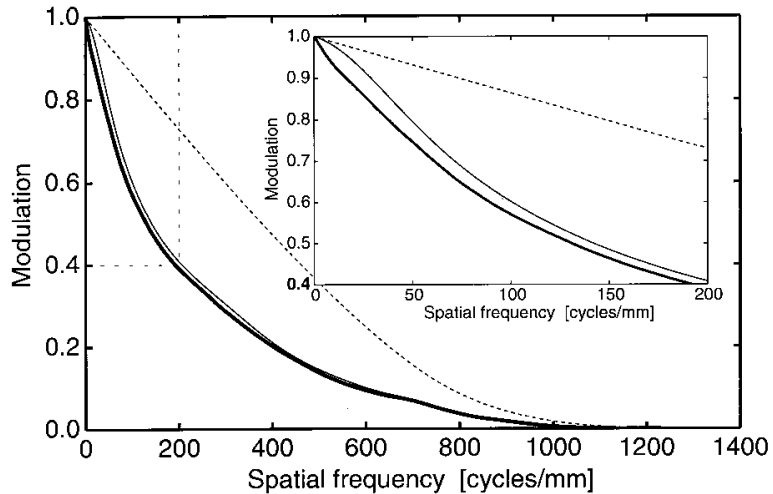

Fig. 5. On-axis polychromatic MTF of the hybrid triplet. Dashed line: diffraction limit. Thin solid line: +1 diffractionorder computation (100\% efficiency assumed for all wavelengths). Thick solid line: zone-decomposition computation. The inset is a magnified view of the low spatial frequency region indicated by the dashed rectangle.

pect of the real PSF far from center, where all diffraction orders interfere in a fairly irregular fashion, compared with the smoother and faster decline of the +1 order PSF. Figure 5 shows the MTF. One may again note the significant departure between the +1 order modeling and our more rigorous model. The absence of a sharp MTF drop at very low spatial frequencies means that higher orders are far from uniform defocusing plateaus, as can be seen from Fig. 4 in which no such structure can be distinguished on the thick solid curve. This rather uncommon behavior is clear from Fig. 2, where it can be seen that the characteristic function of the DOE is mainly intended to correct combined defocus and spherical aberrations rather than pure chromatism.

For comparison purposes, Fig. 6 shows the MTF of an achromatic cemented doublet, with the same focal

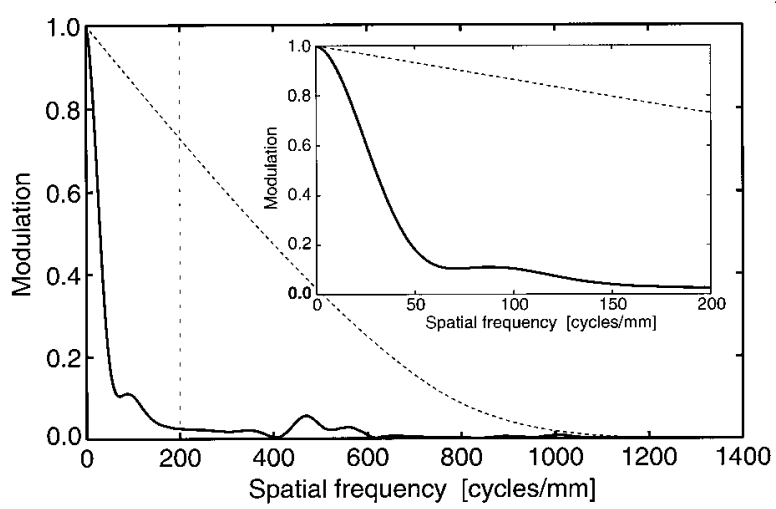

Fig. 6. On-axis polychromatic MTF of an optimized conventional cemented doublet. Dashed line: diffraction limit. Solid line: MTF of a conventional cemented doublet of same focal length and aperture as the hybrid triplet and optimized for polychromatic operation over the same wavelength range $(0.48-0.78 \mu \mathrm{m})$. The inset is a magnified view of the low spatial frequency region indicated by the dashed rectangle. Note that it has a different vertical scale than the one of Fig. 5. 


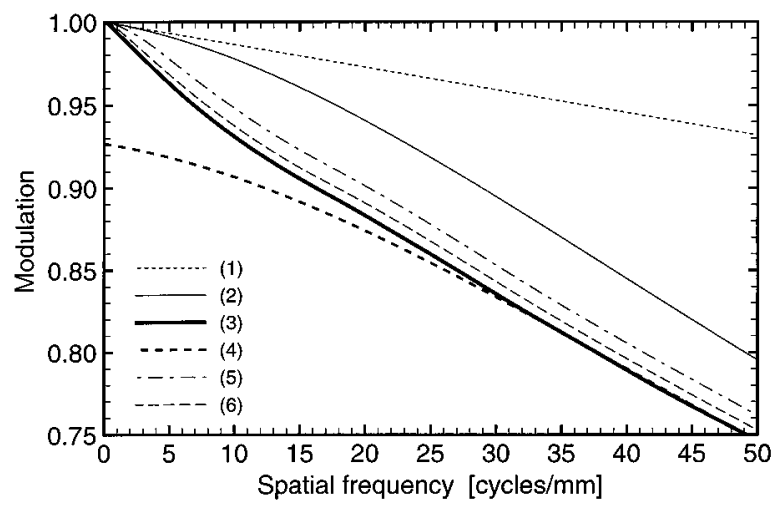

Fig. 7. On-axis polychromatic MTF of the hybrid triplet for low spatial frequencies. (1): diffraction limit. (2): +1 diffraction order (100\% efficiency assumed for all wavelengths). (3): zone decomposition. (4): +1 diffraction order with stray light from nonnominal orders taken into account as a uniform background. (5): incoherent summation of 5 diffraction orders $(-1 \ldots+3)$. (6): incoherent summation of 19 diffraction orders $(-8 \ldots+10)$. See Table 2 for precise definitions of the curves.

length and $f$ number as those of the hybrid triplet, optimized for operation in the same visible-nearinfrared wavelength range. The usefulness of the hybrid design is obvious!

Finally, Fig. 7 shows the polychromatic MTF of the hybrid triplet for low spatial frequencies, as computed from different approximations of the lens behavior. Curves (2) and (3), which correspond to the +1 order and to zone-decomposition computations, respectively, have already been plotted with a different scale on Fig. 5. Curve (4) corresponds to the computation mentioned in the introduction, where the stray light due to nonnominal orders is taken into account through a uniform background. Curves (5) and (6) show the results of the incoherent summation of 5 and 19 orders, respectively, taken symmetrically around the +1 order. Table 2 gives the precise definitions of each computation. For the sake of clarity, the curves for coherent summation of orders are not included; for the present example, they are closed to the incoherent ones. In all cases, the differences to the more rigorous zone decomposition computation [curve (3)] are conspicuous.

Regarding the practical implementation of the zone decomposition for the example curve computations, we extracted the entire hybrid system exit pupil complex amplitude by means of ray tracing with the help of a commercial optical design software. This lead to a discontinuous wave front with a direct relation between step locations and DOE zone boundaries. We thus neglected the Fresnel diffraction that occurred during propagation from the DOE to the exit pupil; this approximation is clearly valid if the DOE is near the exit pupil and seems to be applicable in most practical cases, in the same way as central obscuration of reflecting telescopes are correctly modeled by diffraction PSF or MTF computed by present optical design software. Then, we computed the PSF's and MTF's outside any commercial optical design software, taking advantage of the rotational symmetry of the problem to get the 2D Fourier transform to high precision by means of the more efficient one-dimensional (1D) zero-order Hankel transform ${ }^{6}$ on a piecewise analytical approximation of the discontinuous wave front. Let us emphasize that, with proper care of discontinuities, this zone-decomposition implementation allows for computation of the PSF or the MTF of an entire hybrid system with mainly the same computational effort as the one required for obtaining its +1 diffraction-order PSF or MTF, by avoidance of any explicit computation and coherent summation of the PSF's of each DOE zone. This clearly outperformed,

Table 2. Precise Definitions of the Polychromatic MTF Curves of Fig. 7 and Their Components

Components and

Curve Numbers Definitions

MTF

$\mathrm{OTF}_{m, \lambda}$

$\mathrm{OTF}_{\mathrm{ZD}, \lambda}$

$w_{\lambda}$

$\eta_{m, \lambda}$

Curve 2

Curve 3

Curve 4

Curve 5

Curve 6
Modulus of the complex optical transfer function (OTF), i.e., MTF $=|\mathrm{OTF}|$, where the OTF is the Fourier transform of the real PSF. ${ }^{6}$ The MTF and the OTF are normalized to 1 at the spatial frequency of 0 cycle $/ \mathrm{mm}$.

Monochromatic OTF of the diffraction order $m$ at $\lambda$.

Monochromatic OTF at $\lambda$ for the zone-decomposition computation.

Spectral weights with $\Sigma_{\lambda} w_{\lambda}=1$.

Efficiency of diffraction order $m$ at the wavelength $\lambda$. We have $\sum_{m=-\infty}^{+\infty} \eta_{m, \lambda}=1$ for all $\lambda$. In the case of the assumed perfect continuous DOE etching profile (see Fig. 2), we have $\eta_{m, \lambda}=\operatorname{sinc}^{2}\left(m-\lambda_{\text {Nominal }} / \lambda\right){ }^{7}$ $\mathrm{MTF}_{+1 \operatorname{order}\left(\eta_{1}=100 \% \forall \lambda\right)}=\left|\Sigma_{\lambda} \mathrm{OTF}_{1, \lambda} w_{\lambda}\right|$.

$\mathrm{MTF}_{\mathrm{ZD}}=\left|\Sigma_{\lambda} \mathrm{OTF}_{\mathrm{ZD}, \lambda} w_{\lambda}\right|$.

$\mathrm{MTF}_{+1 \text { order+uniform background }}=\left[\left|\Sigma_{\lambda} \mathrm{OTF}_{1, \lambda} w_{\lambda} \eta_{1, \lambda}\right| / \Sigma_{\lambda} w_{\lambda} \eta_{1, \lambda}\right]\left[\Sigma_{\lambda} w_{\lambda} \eta_{1, \lambda}\right]$, at all nonzero spatial frequencies ( 1 at 0 cycle $/ \mathrm{mm}$ ). Here, because of the nearly optimal choice of $\lambda_{\text {Nominal }}, \Sigma_{\lambda} w_{\lambda} \eta_{1, \lambda}$ reaches 0.927 .

$\mathrm{MTF}_{5 \text { orders }}=\left|\sum_{m=-1}^{+3} \Sigma_{\lambda} \mathrm{OTF}_{m, \lambda} w_{\lambda} \eta_{m, \lambda}\right| / \Sigma_{m=-1}^{+3} \Sigma_{\lambda} w_{\lambda} \eta_{m, \lambda}$, where $\sum_{m=-1}^{+3} \Sigma_{\lambda}$ $w_{\lambda} \eta_{m, \lambda}=0.984$.

$\operatorname{MTF}_{19 \text { orders }}=\left|\Sigma_{m=-8}^{+10} \Sigma_{\lambda} \mathrm{OTF}_{m, \lambda} w_{\lambda} \eta_{m, \lambda}\right| / \Sigma_{m=-8}^{+10} \Sigma_{\lambda} w_{\lambda}\left(\eta_{m, \lambda}\right)$, where $\Sigma_{m=-8}^{+10} \Sigma_{\lambda} w_{\lambda} \eta_{m, \lambda}=0.996$. 
on both precision and computer time, the incoherent or the coherent summation of orders.

\section{Conclusions}

We have proposed to model hybrid systems with a multiaperture approach by coherent summation of the images formed by the various zones of the diffractive component, and we have shown on one example that such rigorous modeling (in the framework of scalar optics) is useful and indeed even necessary for some hybrid systems. In fact, we expect our approach to be especially relevant for hybrid systems in which the DOE has a fairly small number of zones, a highly nonparabolic characteristic function, or nonequal phase discontinuities over the zone boundaries. For the first two cases, the resulting entire PSF significantly departs from the PSF of the nominal order surrounded by weak, large uniform plateaus. This ruins the validity of simple approximations and therefore dictates the use of more rigorous modeling; although both zone decomposition and coherentorder summation are mathematically equivalent, the former is computationally more efficient for the precision usually required. For the last case, no order decomposition is available at all, and the zone decomposition is the only possible approach.

We also have briefly presented the zonedecomposition implementation we used to compute the paper curves. This implementation, based on ray tracing and 1D zero-order Hankel transform, suffers, however, from two limitations: It cannot be entirely performed inside a commercial optical design software, and it is restricted to on-axis images for a rotationally symmetric hybrid system. We are therefore working to implement the zone-decomposition method as addons to commercial software packages with the help of the 2D fast Fourier transform in order to relax the rotational symmetry constraint; however, the accuracy of such an implementation depends on the used sampling grid. This, together with possible application to system design, including optimization, will be the subject of future publications.

\section{References and Notes}

1. T. Stone and N. George, "Hybrid diffractive-refractive lenses and achromats," Appl. Opt. 27, 2960-2971 (1988).

2. M. J. Riedl and J. T. McCann, "Analysis and performance limits of diamond-turned diffractive lenses for the 3-5 and 8-12 micrometer regions," in Infrared Optical Design and Fabrication, R. Hartmann, M. Marietta, and W. J. Smith, eds., Vol. CR38 of SPIE Critical Review Series (SPIE, Bellingham, Wash., 1991), pp. 153-163.

3. M. D. Missig and G. M. Morris, "Diffractive optics applied to eyepiece design," Appl. Opt. 34, 2452-2461 (1995).

4. D. A. Buralli and G. M. Morris, "Effects of diffraction efficiency on the modulation transfer function of diffractive lenses," Appl. Opt. 31, 4389-4396 (1992).

5. M. Born and E. Wolf, Principles of Optics, 6th ed. (Pergamon, Oxford, UK, 1980).

6. J. W. Goodman, Introduction to Fourier Optics, 2nd ed. (McGraw-Hill, New York, 1996).

7. G. J. Swanson, "Binary optics technology: the theory and design of multi-level diffractive optical elements," MIT, Lincoln Lab. Technical Report 854 (Massachusetts Institute of Technology, Lexington, Mass., 1989).

8. H. Sauer, G. Narcy, and P. Chavel, "Kinoform modeling for hybrid optical system design," Diffractive Optics 97, Vol. 12 of Topical Meetings Digests Series (European Optical Society, Orsay, France, 1997), communication D19, pp. 174-175.

9. This is a pure academic exercise, and this layout has been developed independently of the layout of the Melles Griot APO014 Dapromat commercial product that happens to have fairly similar external characteristics. 